\title{
Tridimensional Analysis of Rotatory Subluxation and Sagittal Spinopelvic Alignment in the Setting of Adult Spinal Deformity
}

\author{
Emmanuelle Ferrero, MD ${ }^{\mathrm{a}, \mathrm{b}, *}$, Renaud Lafage, $\mathrm{MS}^{\mathrm{a}}$, Bassel G. Diebo, MD ${ }^{\mathrm{a}}$, \\ Vincent Challier, $\mathrm{MD}^{\mathrm{a}}$, Brice Illharreborde, $\mathrm{MD}, \mathrm{PhD}^{\mathrm{c}}$, Frank Schwab, $\mathrm{MD}^{\mathrm{a}}$, Wafa Skalli, $\mathrm{PhD}^{\mathrm{d}}$, \\ Pierre Guigui, $\mathrm{MD}^{\mathrm{b}}$, Virginie Lafage, $\mathrm{PhD}^{\mathrm{a}}$ \\ a Orthopaedic Surgery, Hospital for Special Surgery, 535 E 70th St, New York, NY 10021, USA \\ ${ }^{\mathrm{b}}$ Orthopaedic Surgery, Hopital Européen Georges Pompidou, 20 Rue Leblanc, 75015 Paris, France \\ ${ }^{\mathrm{c}}$ Orthopaedic Surgery, Robert Debré Hospital, 48 Bd Sérurier, 75019 Paris, France \\ ${ }^{\mathrm{d}}$ Laboratoire de Biomécanique, Arts et Métiers ParisTech, 151 Boulevard de l'Hôpital, 75013 Paris, France
}

\begin{abstract}
Study Design: Retrospective single-center.

Objective: To investigate rotatory subluxation (RS) in adult spinal deformity (ASD) with three-dimensional (3D) stereoradiographic images and analyze relationships between RS, transverse plane parameters, spinopelvic parameters, and clinical outcomes.

Background: Recent research has demonstrated that sagittal plane malalignment and listhesis correlate with ASD patient-reported outcomes. However, there is still a lack of knowledge regarding the clinical impact of 3D evaluation and rotatory subluxation. Recent developments in stereoradiography allow clinicians to obtain full-body standing radiographs with low-dose radiation and 3D reconstruction. Methods: One hundred thirty lumbar ASD patients underwent full-spine biplanar radiographs (EOS Imaging, Paris, France). Clinical outcomes were recorded. Using sterEOS software, spinopelvic parameters and lateral listhesis were measured. 3D transverse plane parameters included apical axial vertebral rotation, axial intervertebral rotation (AIR), and torsion index (sum of AIR in the curve). ASD patients were divided in three groups: AIR $<5^{\circ}, 5^{\circ}<$ AIR $<10^{\circ}$, AIR $>10^{\circ}$. Groups were compared with respect to radiographic and clinical data. Correlations were performed between the transverse and sagittal plane parameters and clinical outcomes.

Results: Patients with AIR $>10^{\circ}$ were significantly older, with larger Cobb angle $\left(39.5^{\circ}\right)$ and greater sagittal plane deformity (pelvic incidence-lumbar lordosis mismatch $11.7^{\circ}$ and pelvic tilt $22.6^{\circ}$ ). The AIR $>10^{\circ}$ group had significantly greater apical vertebra axial rotation apex $\left(24.8^{\circ}\right)$, torsion index $\left(45^{\circ}\right)$, and upper-level AIR $\left(21.5^{\circ}\right)$ than the two other groups. Overall, $27 \%$ of AIR patients did not have two-dimensional (2D) lateral listhesis. Patients with AIR $>10^{\circ}$ had significantly worse Oswestry Disability Index and more low back pain. Conclusion: For patients in which lateral listhesis was unreadable in 2D imaging, rotatory subluxation was revealed using stereoradiography and at an earlier disease stage. Moreover, different 3D transverse plane parameters are related to different patient-reported outcomes. Therefore, axial rotation can be considered in evaluation of lumbar degenerative scoliosis severity and prognosis.
\end{abstract}

Level of Evidence: Level III.

Keywords: Adult spinal deformity; Lumbar scoliosis; Rotatory subluxation; Transverse plane; 3D analysis

Author disclosures: EF (none); RL (none); BGD (none); VC (none); BI (personal fees from Zimmer Spine, other from Implanet, outside the submitted work); FS (personal fees from Nemaris Inc., grants and personal fees from MSD and DePuy, personal fees from K2M and Medicrea, grants from $\mathrm{AO}$, outside the submitted work); WS (none); PG (none); VL (personal fees from Nemaris Inc. and MSD; grants and personal fees from DePuy; grants from ISSG, SRS, and NIH; personal fees from K2M, NuVasive, and Medicrea, outside the submitted work).
The authors acknowledge the French Orthopedic and Traumatologic Surgery Society (SoFCOT), without whose help this research would not have been possible.

*Corresponding author. Hôpital Européen Georges-Pompidou, APHP, Paris V University, 20 rue Leblanc, 75908 Paris Cedex 15, France. Tel.: +331560932 72; fax: +33156092395.

E-mail address: Emmanuelle.ferrero@gmail.com (E. Ferrero). 


\section{Introduction}

In the setting of orthopedic practice, low back pain (LBP) represents roughly $2.5 \%$ of all physician visits [1]. Causes of LBP include but are not limited to scoliosis, adult spinal deformity (ASD), and degenerative spine diseases. With regard to ASD, several authors have investigated the impact of radiographic parameters on health-related quality of life (HRQOL) parameters [2-6]. Radiographic parameters that most highly correlate with patient-reported outcomes are focal (ie, rotatory subluxation [RS]), regional (ie, loss of lordosis), and global (ie, sagittal malalignment) [7]. These complex deformities are often associated with spinal degenerative diseases, such as arthrosis and central or foraminal stenosis, and can lead to pain and radiculopathy [2]. Recently, the literature has also confirmed the impact of spinopelvic alignment on patient reported outcomes [7-9].

Although these studies define the path to an evidencebased approach through the identification of radiographic parameters by correlating them with patient-reported outcomes, they also present certain limitations such as lack of analysis regarding rotatory subluxation and threedimensional (3D) radiographic measurements. In 1981, Perdriolle described scoliosis as a 3D deformity and investigated the transverse plane of this pathology [10]. Although uncommon in the setting of adult pathology, the analysis of the transverse plane in adolescent idiopathic scoliosis revealed that transverse plane parameters are associated with more progressive and severe deformity [10-13]. With the help of numerical models of the spine, some authors also demonstrated that the rotation measured in two-dimensional (2D) on standard radiographs differed from the real 3D rotation $[10,14]$. Taken together, these findings highlight the notion that radiographic measurements only represent a projection of the "true" shape and position of the vertebrae, thus masking a complete understanding of the pathology. In an effort to better understand the spinal deformity and to evaluate the transverse plane, radiograph analyses are often combined with the use of magnetic resonance images or computed tomographic scanner. However, these exams are not performed on patients in a weight bearing position and therefore can lead to failure in identifying patterns of deformity that cause pain. Additionally, use of the computed tomographic scanner is associated with high levels of radiation exposure for patients.

Stereoradiography, which was recently introduced into the clinical practice, allows clinicians to obtain full-body standing biplanar radiographs with low-dose radiation and $3 \mathrm{D}$ reconstructions of the spine with transverse plane analysis $[12,13,15]$. The validity of stereoradiography in routine preoperative and postoperative use has been reported $[15,16]$. However, to our knowledge, few studies, with only small sample sizes, have performed 3D analyses of the spine in ASD with these low-dose biplanar images $[11,17]$.
The purpose of this study is to investigate rotatory subluxation in ASD with low-dose biplanar 3D images and to analyze the relationships between RS, transverse plane parameters, spinopelvic parameters, and patient-reported outcomes. The hypothesis was that stereoradiography could allow the physicians to better describe concealed conditions such as RS and understand potential pain generators that can only be visualized with $3 \mathrm{D}$ imaging.

\section{Material and Methods}

\section{Patient selection}

This study is a single-site retrospective chart review of ASD patients who underwent stereoradiography between November 2012 and July 2014. The study was approved by the institutional review board (IRB) committee. Inclusion criteria were any adult patients older than 18 years with lumbar spinal deformity defined by a coronal Cobb angle greater than $10^{\circ}$ [18]. Exclusion criteria were diagnoses of scoliosis other than degenerative or idiopathic, previous spinal surgery, patients without stereoradiographic images, or images without visible femoral heads or C7.

\section{Data collection}

Standard demographic information was recorded for each patient (age, sex, body mass index [BMI]). HRQOL scores were assessed with a visual analog scale (VAS) and

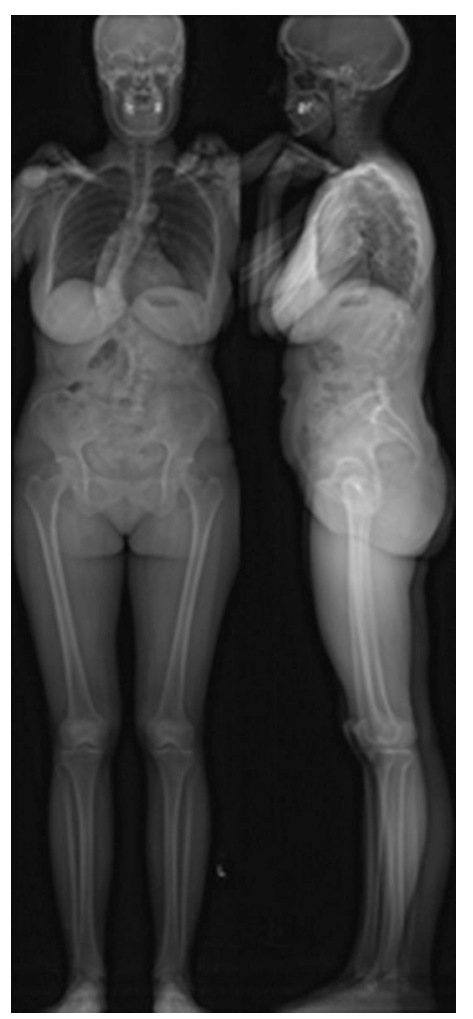

Fig. 1. Stereoradiography imaging coronal and sagittal images. 


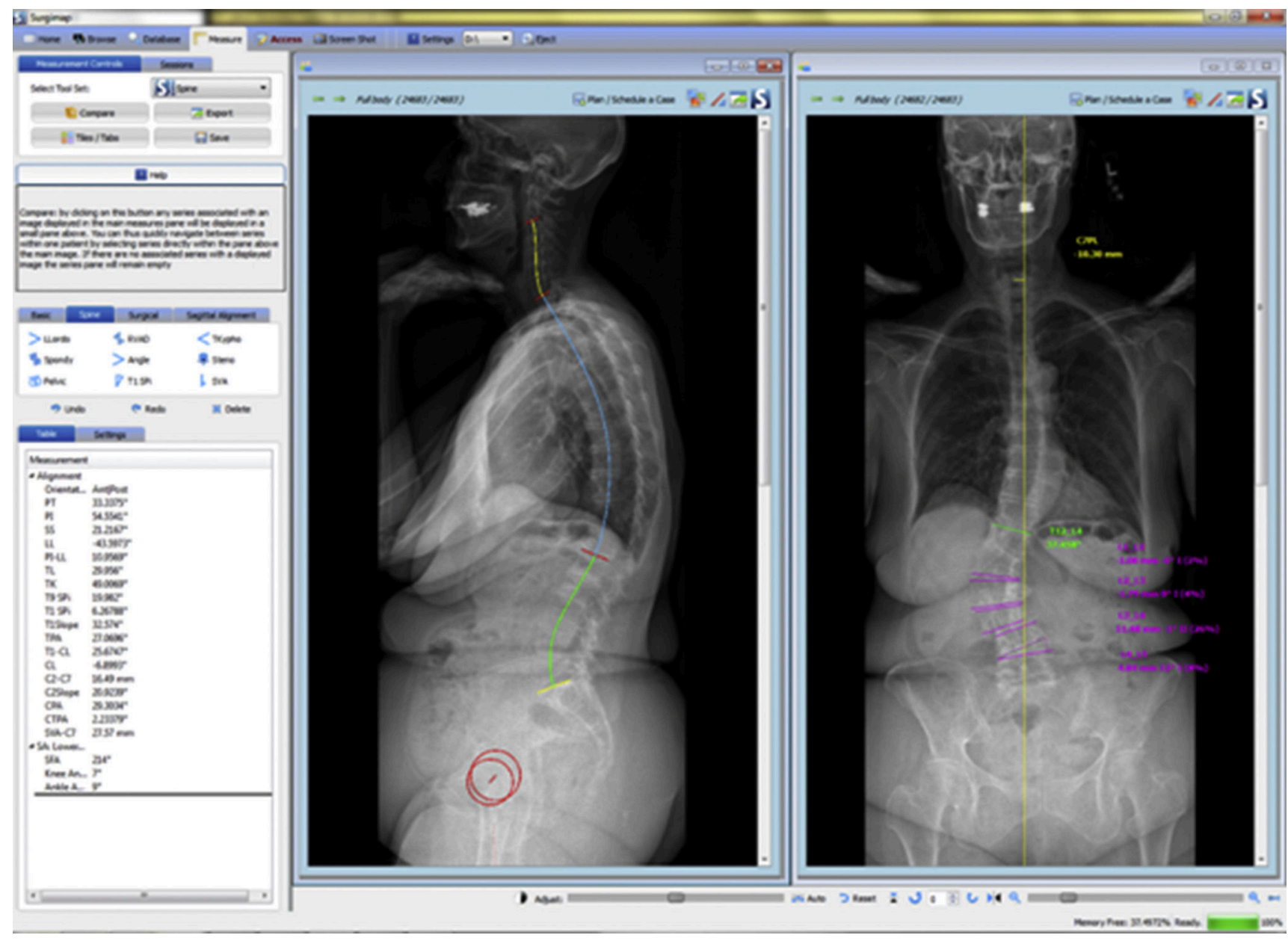

Fig. 2. Example of two-dimensional radiographic measurements.

the Oswestry Disability Index (ODI). Existence of low back pain or radicular leg pain was recorded. Biplanar low-dose stereoradiographs in a standing position were obtained with the EOS system (EOS Imaging, Paris, France) according to a standardized protocol: free-standing position with

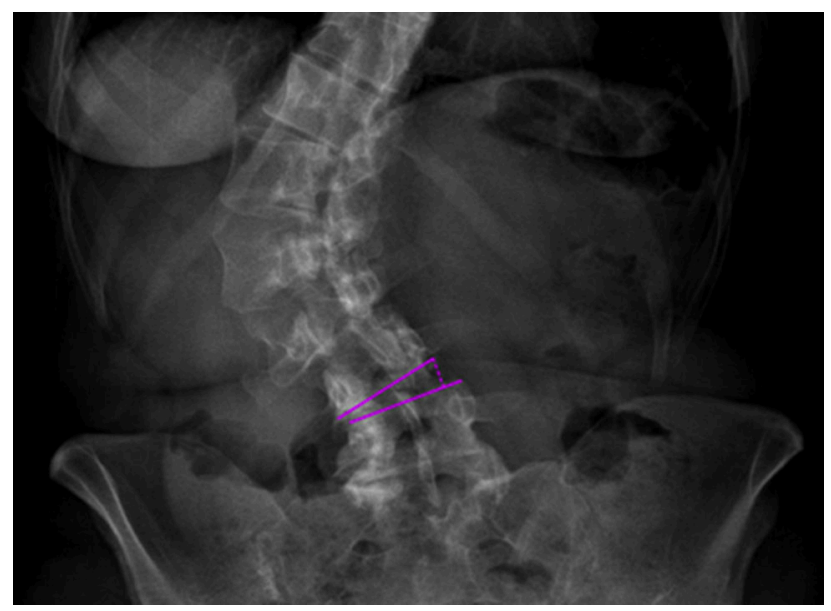

Fig. 3. Evaluation of the lateral listhesis. horizontal gaze, with fists on clavicle to avoid superimposition of the arms with the spine [19-21] (Fig. 1).

A single operator performed the 2D radiographic (Fig. 2) analysis using Surgimap Software (Nemaris Inc., New York). The reproducibility of this software has been validated in a previous study [22]. In $2 \mathrm{D}$, the lateral listhesis was measured at each intervertebral level from the upper to the lower level of the lumbar curve (listhesis) $[23,24]$. Lateral listhesis was measured by the horizontal distance between the superior-lateral corner of the caudal vertebra and the perpendicular to inferior-lateral corner of the cephalad vertebra [7] (Fig. 3). As reported in the literature, rotatory subluxation can be assumed if a lateral listhesis in the coronal plane is greater than $5 \mathrm{~mm}[25,26]$. Thus, existence of lateral listhesis was considered if lateral listhesis was greater than $5 \mathrm{~mm}$.

$3 \mathrm{D}$ reconstructions of the spine and pelvis were obtained by a single operator using SterEOS software 1.2.1 (EOS Imaging), which is based on previously validated software (Fig. 4) $[27,28]$. To correct the effects of a potential axial rotation of the pelvis during the image acquisition, all parameters were expressed in the patients' reference system based on a vertical plane passing through the center of the acetabulum [29]. In 3D, 


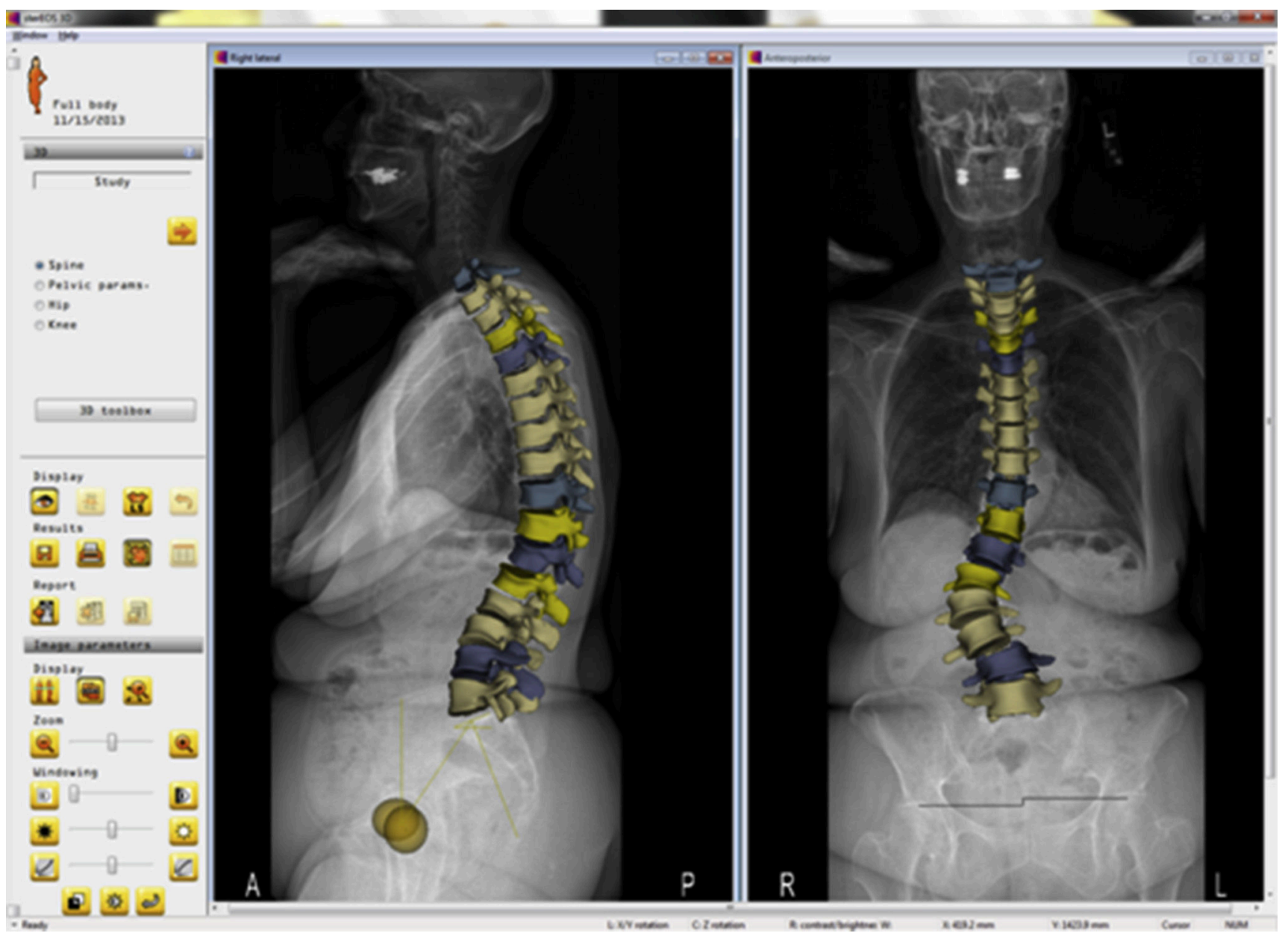

Fig. 4. Example of three-dimensional radiographic reconstruction.
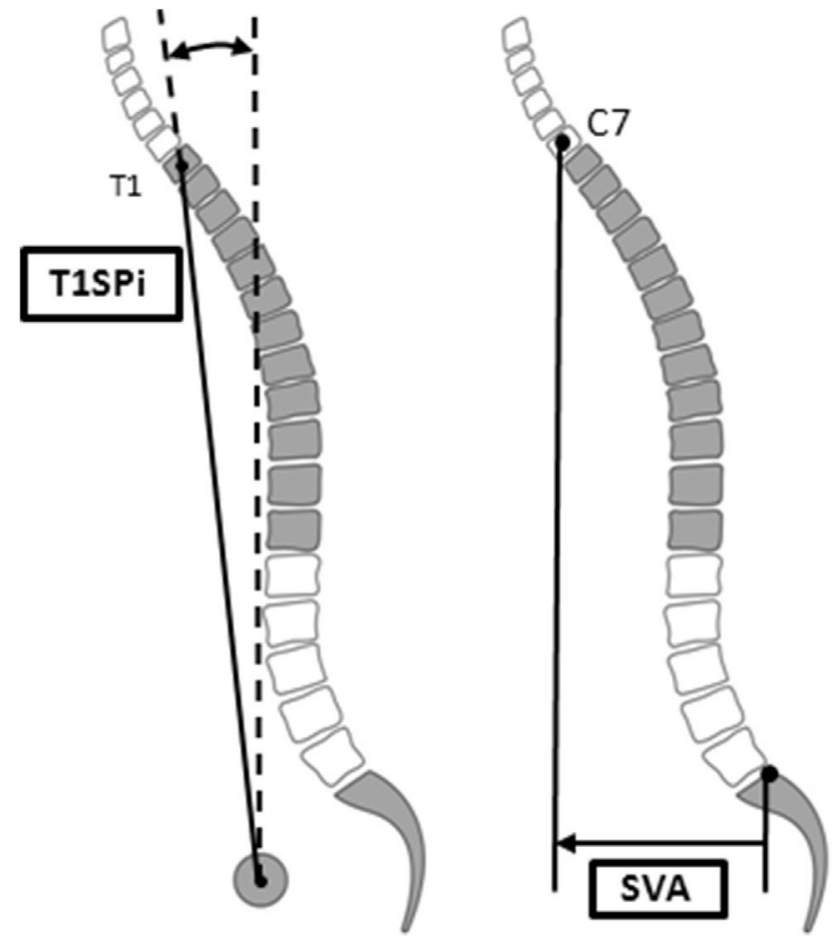

Fig. 5. Global sagittal measurements. the sagittal plane was evaluated in terms of global alignment (sagittal vertical axis [SVA], T1 spinopelvic inclination [T1SPi]; Fig. 5), pelvic parameters (pelvic incidence [PI], sacral slope [SS], and pelvic tilt [PT]), and spinal curvatures (T1-T12 kyphosis, L1-S1 lordosis, and PI minus lumbar lordosis [LL] mismatch [PI-LL]) [30,31]. The coronal plane was described by the main Cobb angle [Cobb] and the coronal

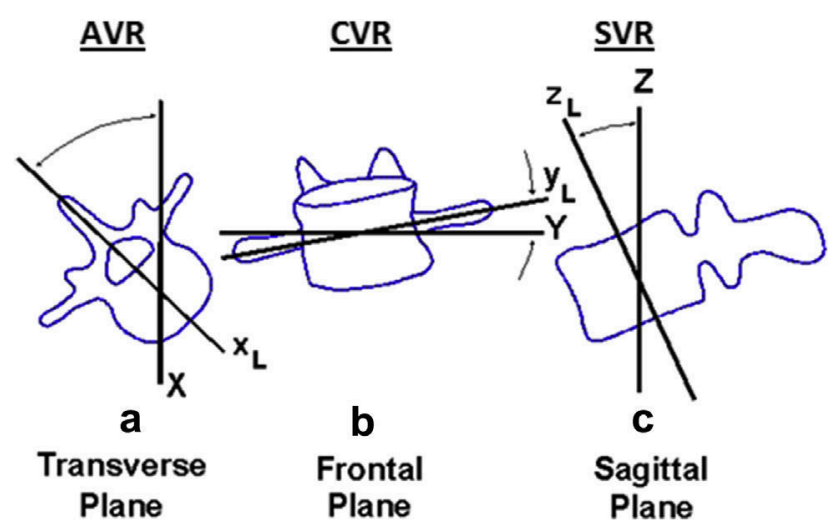

Fig. 6. Axial (AVR), sagittal (SVR), and coronal (CVR) vertebral rotation. 


\section{Coronal Curve Types}

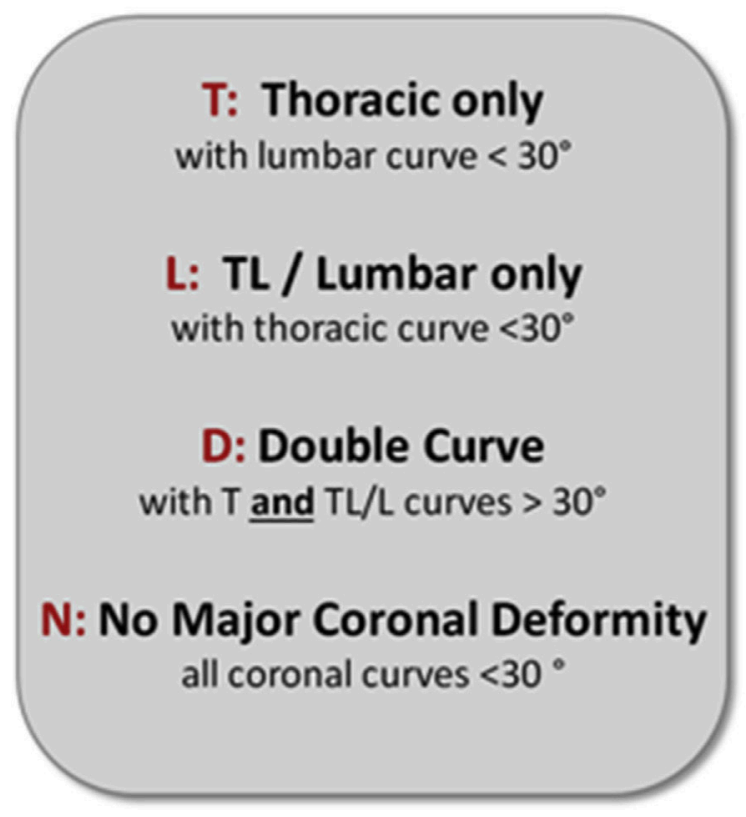

Sagittal Modifiers

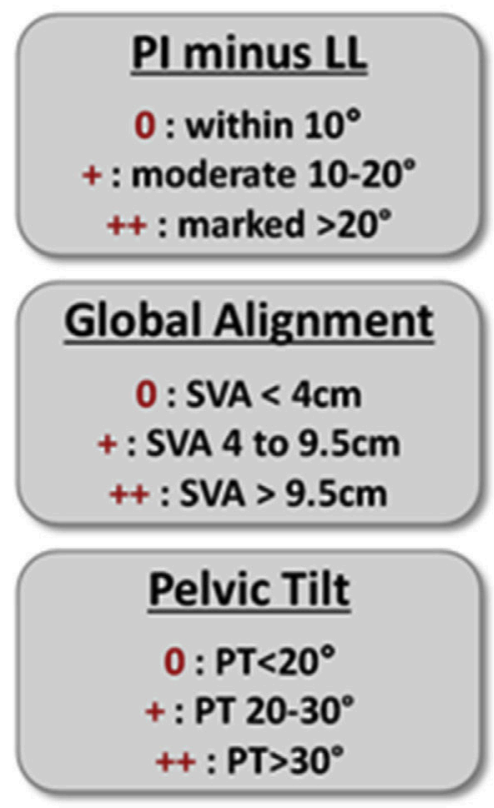

Fig. 7. SRS-Schwab classification.

C7 plumb line [C7PL]. The 3D vertebral and intervertebral rotations were expressed in the axial, frontal, and sagittal planes (Fig. 6). Transverse-plane parameters were quantified and included the apical vertebra axial rotation (AVR apex), the axial intervertebral rotation of the upper and lower levels of the main curve (upper AIR, lower AIR), and the maximal axial intervertebral rotation (AIR max). Axial intervertebral rotation corresponded to the axial rotation of the upper vertebra in the plane of the lower vertebra. The torsion index of the main lumbar curve, described by Steib as the sum of the axial intervertebral rotation of the curve, was calculated [11]. Finally, the SRS-Schwab classification was applied to describe the curve types: a curve type $\mathrm{T}$ was defined as a thoracic major curve of greater than $30^{\circ}$ and apical level of T9 or higher; a curve type L was defined as a lumbar or thoracolumbar major curve greater than $30^{\circ}$ and an apical level of T10 or lower; a curve type D was defined as a double major curve, with each curve greater than $30^{\circ}$; a curve type $\mathrm{N}$ was defined as a coronal curve not greater than $30^{\circ}$ (Fig. 7) [32].

\section{Stratification by group}

In mild to severe scoliosis, the mean error for axial rotation measurement with stereoradiographic images has been reported between $5^{\circ}$ and $10^{\circ}$ [15,27]. Thus, ASD patients were divided into three groups: AIR below $5^{\circ}$, AIR within $5^{\circ}$ to $10^{\circ}$, and AIR above $10^{\circ}$.

\section{Statistical analysis}

After evaluating the normal distribution of the parameters with a Shapiro-Wilk test, descriptive analysis was conducted using means and standard deviations for continuous variables, and frequency analyses for categorical variables.

The three groups of patients were compared in terms of demographic and radiographic parameters. Group comparison was achieved with a chi-square test for categorical variables, an analysis of variance for normal continuous variables, or Kruskal-Wallis test if they were not normally distributed. Finally, after a descriptive analysis of patient-reported outcomes, the correlation

Table 1

Demographic parameters and types of curves in the AIR groups.

\begin{tabular}{|c|c|c|c|c|}
\hline $\begin{array}{l}\text { Demographic } \\
\text { parameters } \\
\text { and curves } \\
\text { characteristics }\end{array}$ & $\begin{array}{l}\text { AIR }>10^{\circ} \\
(\mathrm{n}=78)\end{array}$ & $\begin{array}{l}5^{\circ}<\mathrm{AIR}<10^{\circ} \\
(\mathrm{n}=31)\end{array}$ & $\begin{array}{l}\text { AIR }<5^{\circ} \\
(\mathrm{n}=21)\end{array}$ & $\mathrm{p}$ \\
\hline $\begin{array}{l}\text { Age, years, } \\
\text { mean }(\mathrm{SD})\end{array}$ & $61.5 *(15.1)$ & $55.2(20.3)$ & $48(22.1)$ & .007 \\
\hline BMI, mean (SD) & $25.4(6.3)$ & $25.2(5.1)$ & $24.5(5.3)$ & .81 \\
\hline $\begin{array}{l}\text { Sex (female, } \%) \\
\text { Curve type, n (\%) }\end{array}$ & $90-$ & $81-$ & $62 * \quad-$ & .01 \\
\hline $\mathrm{D}$ & $32(41)$ & $5(16)$ & $3(14)$ & .001 \\
\hline & $24(31)$ & $5(16)$ & $5(24)$ & \\
\hline $\mathrm{N}$ & $22(28)$ & $21(68)$ & $13(62)$ & \\
\hline $\begin{array}{l}\text { Lateral listhesis } \\
\qquad>5 \mathrm{~mm}, \mathrm{n}(\%)\end{array}$ & $61(78)$ & $12(39)$ & $6(29)$ & .001 \\
\hline
\end{tabular}

AIR, axial intervertebral rotation; BMI, body mass index.

* Significant difference with the two other groups.

Bold indicate significant $\mathrm{p}$ values. 
Table 2

Radiographic parameters of the AIR groups.

\begin{tabular}{|c|c|c|c|c|c|c|c|}
\hline \multirow[t]{2}{*}{ Radiographic parameters } & \multicolumn{2}{|c|}{ AIR $>10^{\circ}(\mathrm{n}=78)$} & \multicolumn{2}{|c|}{$5^{\circ}<\operatorname{AIR}<10^{\circ}(\mathrm{n}=31)$} & \multicolumn{2}{|c|}{$\operatorname{AIR}<5^{\circ}(\mathrm{n}=21)$} & \multirow[t]{2}{*}{$\mathrm{p}$} \\
\hline & Mean & SD & Mean & SD & Mean & $\mathrm{SD}$ & \\
\hline Lumbar Cobb $\left(^{\circ}\right)$ & $39.5^{*}$ & 15.5 & 24.3 & 11.5 & 22.9 & 8.1 & .001 \\
\hline SVA (mm) & 39.2 & 62.9 & 25.2 & 47.9 & 9.0 & 44.2 & .08 \\
\hline PI minus LL $\left(^{\circ}\right)$ & $11.7^{*}$ & 23.2 & 4.5 & 18.2 & 2.1 & 18.9 & .024 \\
\hline Pelvic tilt $\left({ }^{\circ}\right)$ & $22.6^{\dagger}$ & 12 & 17.3 & 9.9 & $15.0^{\dagger}$ & 11.8 & .01 \\
\hline $\operatorname{T} 1 \mathrm{SPi}\left({ }^{\circ}\right)$ & -2.5 & 6.9 & -2.8 & 5.2 & -4.4 & 4.2 & .46 \\
\hline $\mathrm{L} 1-\mathrm{S} 1\left(^{\circ}\right)$ & 41.1 & 19.9 & 46.7 & 20.6 & 51.1 & 16.3 & .08 \\
\hline PI $\left(^{\circ}\right)$ & 52.8 & 13.7 & 51.1 & 14.2 & 49.0 & 14.5 & .52 \\
\hline AVR apex $\left(^{\circ}\right)$ & $24.8^{*}$ & 14.8 & 9.9 & 5.9 & 7.0 & 3.8 & .001 \\
\hline Torsion index $\left({ }^{\circ}\right)$ & $45.0^{*}$ & 27.6 & 16.1 & 6.4 & 10.8 & 5.6 & .001 \\
\hline $\operatorname{AIR} \max \left({ }^{\circ}\right)$ & $21.5^{*}$ & 10.7 & 8.2 & 1.5 & 4.3 & 1.3 & .001 \\
\hline Upper AIR $\left({ }^{\circ}\right)$ & $7.8^{*}$ & 6.0 & 3.4 & 3.0 & 2.4 & 1.9 & .001 \\
\hline
\end{tabular}

AIR max, maximal axial intervertebral rotation; AVR apex, apical vertebra axial rotation; C7PL, C7 plumb line; LL, lumbar lordosis; PI, pelvic incidence; SD, standard deviation; SVA, sagittal vertical axis; T1SPi, T1 spinopelvic inclination.

* Significant difference with respect to the two other groups.

$\dagger$ Significant difference between the two groups.

Bold indicate significant $\mathrm{p}$ values.

between outcomes and radiographic data was computed. The level of significance was set at $p<.05$. The statistical analysis was performed in Stata, version 13.0 (Statacorp, College Station, TX).

\section{Results}

\section{Demographic}

One hundred thirty patients met the inclusion criteria; all of them had 3D reconstructions. Eighty-three percent of patients were female $(n=108)$, with a mean age of $57.6 \pm$ 18.3 years, and a mean BMI of $25.2 \pm 5.9$. The distribution of SRS-Schwab coronal types was as follows: $\mathrm{L}=34$ (26\%), $\mathrm{D}=40(31 \%)$, and $\mathrm{N}=56(43 \%)$.

Patients with AIR $>10^{\circ}$ were significantly older than patients without AIR; there was no significant difference in terms of BMI between groups. Female gender was significantly less frequent in the AIR $<5^{\circ}$ group. In terms of curve types, there was a majority of $\mathrm{D}$ and $\mathrm{L}$ curves in the AIR $>10^{\circ}$ group, whereas $\mathrm{N}$ curves were more common in the $5^{\circ}<$ AIR $<10^{\circ}$ group and the AIR $<5^{\circ}$ group. Seventy-nine $(61 \%)$ patients had at least one lateral listhesis greater than $5 \mathrm{~mm}$ in the coronal plane. Patients in the AIR $>10^{\circ}$ group had significantly more lateral listhesis than the other two groups (Table 1).

\section{Descriptive radiographic analysis}

The analysis revealed a mean Cobb angle of $33.2^{\circ} \pm$ $15.6^{\circ}$ and a mean AVR apex of $18.3^{\circ} \pm 14.3^{\circ}$. The Cobb angle was significantly larger in the AIR $>10^{\circ}$ group. No significant differences were found in coronal $\mathrm{C} 7$ plumb line. Among the 78 patients with AIR $>10^{\circ}, 17$ did not have listhesis, 23 had one level of listhesis, 23 had two levels, 14 had three levels, and 1 had four levels. Among the 31 patients with $5^{\circ}<$ AIR $<10^{\circ}, 19$ did not have listhesis, 9 had one level of listhesis, and 3 had two levels. Among the 21 patients with $\mathrm{AIR}<5^{\circ}, 15$ did not have listhesis, and 6 patients had one or two levels of listhesis. Thus, $27 \%$ of the patients with AIR greater than $5^{\circ}$ and $10 \%$ of the patients with AIR greater than $10^{\circ}$ did not have lateral listhesis

Table 3

Pearson correlations between EOS parameters $(\mathrm{p}<.05)$.

\begin{tabular}{|c|c|c|c|c|c|c|c|c|c|}
\hline & Cobb angle & AVR apex & AIR max & TI & PT & PI-LL & SVA & T1SPi & $\overline{\mathrm{T} 1-\mathrm{T} 12}$ \\
\hline Cobb angle & 1.000 & & & & & & & & \\
\hline AVR apex & 0.697 & 1.000 & & & & & & & \\
\hline AIR max & 0.670 & 0.789 & 1.000 & & & & & & \\
\hline PT & - & 0.286 & 0.429 & 0.408 & 1.000 & & & & \\
\hline PI-LL & 0.259 & 0.419 & 0.496 & 0.474 & 0.745 & 1.000 & & & \\
\hline SVA & 0.190 & 0.294 & 0.414 & 0.345 & 0.444 & 0.713 & 1.000 & & \\
\hline
\end{tabular}

AIR max, maximal axial intervertebral rotation; AVR apex, apical vertebra axial rotation; LL, lumbar lordosis; PI, pelvic incidence; PT, pelvic tilt; SVA, sagittal vertical axis; TI, Torsion Index; T1SPi, T1 spinopelvic inclination. 
Table 4

Clinical outcomes comparison between groups with or without AIR.

\begin{tabular}{|c|c|c|c|c|c|c|c|c|}
\hline \multirow[t]{2}{*}{ Clinical outcomes } & \multirow[t]{2}{*}{$\mathrm{n}$} & \multicolumn{2}{|c|}{ AIR $>10^{\circ}(\mathrm{n}=78)$} & \multicolumn{2}{|c|}{$5^{\circ}<\mathrm{AIR}<10^{\circ}(\mathrm{n}=31)$} & \multicolumn{2}{|c|}{$\operatorname{AIR}<5^{\circ}(\mathrm{n}=21)$} & \multirow[t]{2}{*}{$\mathrm{p}$} \\
\hline & & $\mathrm{n}$ & Mean $(\mathrm{SD})$ or $\mathrm{n}(\%)$ & $\mathrm{n}$ & Mean (SD) or $\mathrm{n}(\%)$ & $\mathrm{n}$ & Mean (SD) or $\mathrm{n}(\%)$ & \\
\hline ODI & 56 & 31 & $36.3(22.8)$ & 16 & $33.2(20.9)$ & 9 & $16 *(16)$ & .03 \\
\hline VAS & 119 & 72 & $5.1(2.5)$ & 29 & $4.7(2.3)$ & 18 & $3.9(3.1)$ & .29 \\
\hline Leg pain & 119 & 72 & $44(56 \%)$ & 29 & $16(52 \%)$ & 18 & $10(58 \%)$ & .74 \\
\hline LBP & 119 & 72 & $64(90 \%)$ & 29 & $26(84 \%)$ & 18 & $10(58 \%)^{*}$ & .01 \\
\hline
\end{tabular}

AIR, axial intervertebral rotation; LBP, low back pain; ODI, Oswestry Disability Index; SD, standard deviation; VAS, visual analog scale.

* Significant difference with the two other groups.

Bold indicate significant $p$ values.

visible on the coronal plane on 2D images. Overall, the most common listhesis level was L3-L4 (33\%).

Patients with AIR $>10^{\circ}$ had significantly larger sagittal deformity in terms of loss of lumbar lordosis (PI-LL) and pelvic retroversion (PT). They also exhibited a greater transverse plane deformity with greater AVR apex, torsion index, AIR max, and upper AIR than the two other groups (Table 2).

\section{Correlation across radiographic parameters}

For the entire set of patients, the number of levels with AIR greater than $5^{\circ}$ was significantly correlated with all the sagittal modifiers (PI-LL: $r=0.440, P T: ~ r=0.314$, and SVA: $r=0.399, p<.05)$, as well as the number of AIR greater than $10^{\circ}$ (PI-LL: $\mathrm{r}=0.452$, PT: $\mathrm{r}=0.422$, and SVA: $r=0.323, p<.05)$. Significant correlations were identified between transverse plane parameters and the three SRS-Schwab sagittal modifiers. T1SPi significantly correlated with the Cobb angle, AVR apex, max AIR, and torsion index. As expected, the torsion index was significantly correlated with the AVR apex $(r=0.641, \mathrm{p}<.05)$. The Cobb angle was significantly correlated with AVR apex, AIR max, and the torsion index $(\mathrm{r}>0.6, \mathrm{p}<$ .05) (Table 3).

\section{Clinical parameters}

ODI score was available for 56 patients. The AIR $<5^{\circ}$ group had a lower ODI score than the $5^{\circ}<$ AIR $<10^{\circ}$ and AIR $>10^{\circ}$ groups. The incidence of leg pain $(56 \%$ in

Table 5

Cobb angle and AVR in recent scoliosis study with EOS Imaging.

\begin{tabular}{lllll}
\hline Literature & $\begin{array}{l}\text { No. of } \\
\text { patients }\end{array}$ & $\begin{array}{l}\text { Scoliosis } \\
\text { type }\end{array}$ & $\begin{array}{l}\text { Mean Cobb } \\
\text { angle }\left(^{\circ}\right)\end{array}$ & $\begin{array}{l}\text { AVR } \\
\text { apex }\left(^{\circ}\right)\end{array}$ \\
\hline Current study & 130 & ASD & 33.2 & 18.3 \\
Steib [11] & 10 & ASD & 50.0 & 24.0 \\
Dubousset [12] & 45 & AIS & 61.1 & 19.9 \\
Ilharreborde [15] & 24 & AIS & 62.0 & 21.0 \\
Courvoisier [13] & 33 & AIS & 16.0 & 9.0 \\
& 45 & AIS & 13.0 & 4.0 \\
Gille [16] & 30 & AIS & 16.0 & 1.2 \\
\hline
\end{tabular}

ASD, adult spinal deformity; AIS, adolescent idiopathic scoliosis; AVR apex, apical vertebra axial rotation. the AIR $>10^{\circ}$ group) was not significantly different between the three groups. Low back pain was significantly more frequent in patients with AIR $>10^{\circ}$ or with $5^{\circ}<$ AIR $<10^{\circ}(90 \%$ vs. $58 \%$ and $84 \%$ vs. $58 \%$ $\mathrm{p}=.01)$ (Tables 4 and 5).

For the entire set of patients, the number of levels with AIR greater than $5^{\circ}$ was significantly correlated with ODI $(\mathrm{r}=0.402, \mathrm{p}<.05)$. There was no significant correlation between 3D transverse plane parameters and ODI. SVA was correlated to ODI $(\mathrm{r}=0.473, \mathrm{p}<.05)$.

\section{Discussion}

The aim of this study was to investigate rotatory subluxation in ASD and analyze the relationships between rotatory subluxation, spinopelvic parameters, and patientreported outcomes. Results demonstrated that patients with larger AIR had more lateral listhesis, greater transverse plane deformity, and greater sagittal deformity. Significant differences existed between groups in terms of clinical parameters. As a matter of fact, the current study was also a way to analyze the clinical relevance of 3D analysis in ASD.

\section{Evaluation of rotatory subluxation and listhesis}

This study demonstrated that patients with AIR $>10^{\circ}$ had a significantly greater transverse plane deformity with greater apical axial vertebral rotation, maximal intervertebral rotation and torsion index (Table 2). An important finding in this study is that $27 \%$ of the patients with AIR greater than $5^{\circ}$ and $10 \%$ of the patients with AIR greater than $10^{\circ}$ did not have lateral listhesis visible on the coronal plane. These results confirmed that lateral listhesis is not easily readable on $2 \mathrm{D}$ radiographs and can remain unnoticed until it reaches a severe stage. On the other hand, more information is provided by measuring axial intervertebral rotation in 3D.

Regarding measurements of rotation, Cobb, Nash and Moe, Perdriolle, and others have described several methods using standard coronal radiographs $[10,33,34]$; a mean error of about $5^{\circ}$ for mild scoliosis has been reported [34-38]. As demonstrated by Skalli et al. [14], one of the limitations of these methods relates to the fact that they do not take into account the impact of the vertebral rotations in the other 

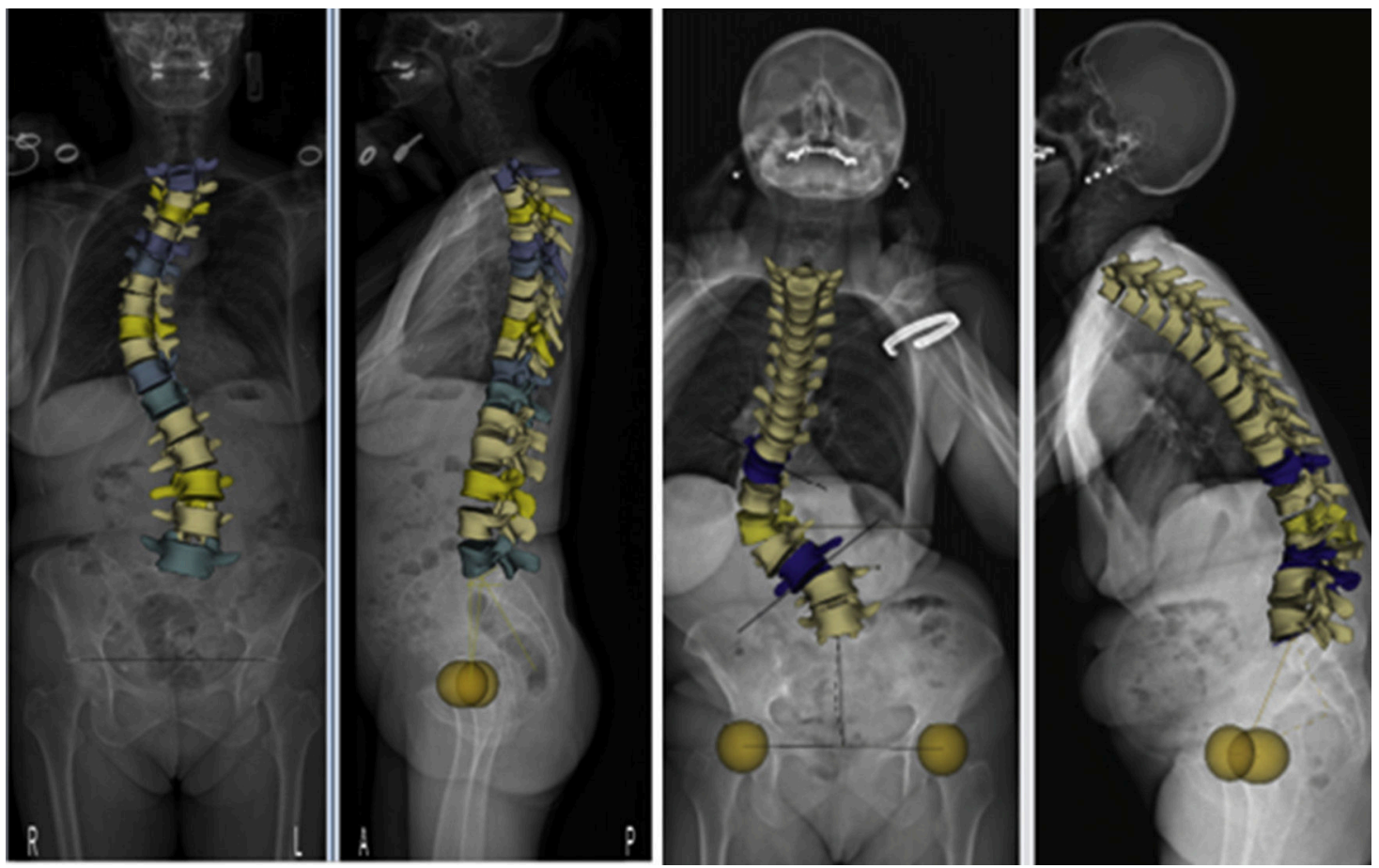

Fig. 8. Examples of patients with different amounts of axial intervertebral rotation (AIR) and lateral listhesis.

planes. With the biplanar system, rotation of each vertebra and of every vertebral segment can be measured in the three planes accurately with an error estimation of only $\pm 1.6^{\circ}$ for coronal rotation, $\pm 2.0^{\circ}$ for sagittal rotation, and $\pm 5^{\circ}$ for axial rotation $[11-13,16,20]$.

\section{Tridimensional characterization of degenerative scoliosis}

In the current study, transverse plane parameters correlated with Cobb (Fig. 8). In addition, a significant correlation was found between the Cobb angle and axial apical rotation. When comparing our results with the literature (Table 5), it seems that adult patients with lumbar scoliosis exhibited the same axial apical rotation than AIS patients, despite a small coronal Cobb angle. Of note, although Dubousset et al. reported a torsion index of $15.8^{\circ}$ in a cohort of AIS patients with a Cobb angle of $61.2^{\circ}$, the ASD patients in the AIR $>10^{\circ}$ group had a torsion index of $45^{\circ}$ [12]. A new finding in this study is the significant correlation between transverse plane parameters and SRS sagittal modifiers.

\section{Relationship between radiographic and clinic parameters}

The current study assessed the relationship between 3D measurements and clinical data. Frequency of low back pain in patients with ASD and rotatory subluxation has been reported as high as 80 percent [39]. In an ASD radiographic study, Marty-Poumarat reported similar outcomes, with $84 \%$ of patients reporting LBP, $43 \%$ radicular leg pains, and the majority having lateral listhesis at the L3-L4 level (66\%). However, she did not establish any correlation between patient-reported outcomes and radiographic parameters [40].

Previously, Ploumis found increased LBP in patients with lateral listhesis but without any correlation with ODI [26]. Interestingly, in the current study, the number of levels with AIR significantly correlated with ODI $(r=0.402, \mathrm{p}<$ .05). Moreover, significant correlations were observed between 3D axial rotation parameters (ie, torsion index, maximal intervertebral axial rotation) and VAS or LBP. Significant differences between the three groups in terms of LBP could be explained by the high rotatory constraint on intervertebral disc resultant in disc degeneration with LBP and nerve roots compression due to listhesis. As rotatory subluxation corresponds not only to the lateral listhesis but also to axial rotation, these results emphasize the importance of 3D axial parameters in assessing the consequences of rotatory subluxation on ASD patient-reported outcomes.

\section{Limitations and future directions}

One advantage of the biplanar system used in this study is that it provides a patient's specific full body reconstruction in a functional standing position, thereby allowing a surgeon to analyze compensatory mechanisms. However, these benefits may be dampened by bone quality and visualization issues. Indeed, analysis of concealed anatomic details (such as facet joints) was difficult in ASD patients with large Cobb angles and poor bone quality. A recent 
study on ASD patients reported 3D measurements' reproducibility and accuracy with stereoradiography [28]. This study highlights that $3 \mathrm{D}$ reconstructions are less accurate in terms of axial rotations for patients with larger Cobb angles than those with beginning degenerative scoliosis. Few HRQOL scores were available, which limits our ability to fully capture the clinical impact of rotatory subluxation. Moreover, progression of pathology was not fully studied and is the basis for an ongoing study based on transverse plane parameters.

\section{Conclusion}

In the current study, clinical relevance of ASD 3D analysis was emphasized. This study utilizes 3D stereoradiography imaging to identify rotatory subluxation with transverse plane parameters as an important driver of pain and disability in ASD patients. Axial intervertebral rotation was correlated with previously established SRS-Schwab modifiers. Patients with larger axial intervertebral rotation had worst clinical scores. Analyzing axial rotation using 3D imaging may predict pathology at earlier stages than traditional listhesis measurement in $2 \mathrm{D}$ radiographs. Overall, axial rotation may prove to be an important factor to consider when evaluating ASD patients.

\section{References}

[1] Deyo RA, Mirza SK, Martin BI. Back pain prevalence and visit rates: estimates from U.S. national surveys. Spine (Phila Pa 1976) 2002;31: 2724-7.

[2] Liu W, Chen X, Jia L, et al. The clinical features and surgical treatment of degenerative lumbar scoliosis: a review of 112 patients. Orthop Surg 2009;1:176-83.

[3] Glassman SD, Berven S, Bridwell K, et al. Correlation of radiographic parameters and clinical symptoms in adult scoliosis. Spine (Phila Pa 1976) 2005;30:682-8.

[4] Glassman SD, Bridwell K, Dimar JR, et al. The impact of positive sagittal balance in adult spinal deformity. Spine (Phila Pa 1976) 2005;30:2024-9.

[5] Lafage V, Schwab F, Patel A, et al. Pelvic tilt and truncal inclination: two key radiographic parameters in the setting of adults with spinal deformity. Spine (Phila Pa 1976) 2009;34:E599-606.

[6] Schwab F, Dubey A, Gamez L, et al. Adult scoliosis: prevalence, SF-36, and nutritional parameters in an elderly volunteer population. Spine (Phila Pa 1976) 2005;30:1082-5.

[7] Schwab F, Farcy J, Bridwell K, et al. A clinical impact classification of scoliosis in the adult. Spine (Phila Pa 1976) 2006;31: 2109-14.

[8] Schwab FJ, Smith VA, Biserni M, et al. Adult scoliosis: a quantitative radiographic and clinical analysis. Spine (Phila Pa 1976) 2002;27: 387-92.

[9] Glassman SD, Hamill CL, Bridwell KH, et al. The impact of perioperative complications on clinical outcome in adult deformity surgery. Spine (Phila Pa 1976) 2007;32:2764-70.

[10] Perdriolle R, Vidal J. A study of scoliotic curve. The importance of extension and vertebral rotation [in French]. Rev Chir Orthop Reparatrice Appar Mot 1981;67:25-34.

[11] Steib J, Dumas R, Mitton D, et al. Surgical correction of scoliosis by in situ contouring: a detorsion analysis. Spine (Phila Pa 1976) 2004;29:193-9.
[12] Dubousset J, Ilharreborde B, Le Huec J-C. Use of EOS imaging for the assessment of scoliosis deformities: application to postoperative 3D quantitative analysis of the trunk. Eur Spine J 2014;23(Suppl 4): S468.

[13] Courvoisier A, Drevelle X, Dubousset J, et al. Transverse plane 3D analysis of mild scoliosis. Eur Spine J 2013;22:2427-32.

[14] Skalli W, Lavaste F, Descrimes JL. Quantification of threedimensional vertebral rotations in scoliosis: what are the true values? Spine (Phila Pa 1976) 1995;20:546-53.

[15] Ilharreborde B, Steffen JS, Nectoux E, et al. Angle measurement reproducibility using EOS three-dimensional reconstructions in adolescent idiopathic scoliosis treated by posterior instrumentation. Spine (Phila Pa 1976) 2011;36:E1306-13.

[16] Gille O, Champain N, Benchikh-El-Fegoun A, et al. Reliability of 3D reconstruction of the spine of mild scoliotic patients. Spine (Phila Pa 1976) 2007;32:568-73.

[17] Steffen J-S, Obeid I, Aurouer N, et al. 3D postural balance with regard to gravity line: an evaluation in the transversal plane on 93 patients and 23 asymptomatic volunteers. Eur Spine J 2010;19:760-7.

[18] Schwab FJ, Blondel B, Bess S, et al. Radiographical spinopelvic parameters and disability in the setting of adult spinal deformity: a prospective multicenter analysis. Spine (Phila Pa 1976) 2013;38:E803-12.

[19] Faro FD, Marks MC, Pawelek J, et al. Evaluation of a functional position for lateral radiograph acquisition in adolescent idiopathic scoliosis. Spine (Phila Pa 1976) 2004;29:2284-9.

[20] Kalifa G, Charpak Y, Maccia C, et al. Evaluation of a new low-dose digital X-ray device: first dosimetric and clinical results in children. Pediatr Radiol 1998;28:557-61.

[21] Dubousset J, Charpak G, Skalli W, et al. Système EOS: la radiographie de la tête aux pieds face et profil simultanés à très basses doses de radiations. Rev Chir Orthop Reparatrice Appar Mot 2007;93: $141-3$.

[22] Lafage R, Ferrero E, Henry JK, et al. Validation of a new computerassisted tool to measure spinopelvic parameters. Spine J 2015;15: 2493-502.

[23] Legaye J, Duval-Beaupère G, Hecquet J, et al. Pelvic incidence: a fundamental pelvic parameter for three-dimensional regulation of spinal sagittal curves. Eur Spine J 1998;7:99-103.

[24] Vialle R, Levassor N, Rillardon L, et al. Radiographic analysis of the sagittal alignment and balance of the spine in asymptomatic subjects. J Bone Joint Surg Am 2005;87:260-7.

[25] Ploumis A, Transfeldt EE, Gilbert TJJ, et al. Degenerative lumbar scoliosis: radiographic correlation of lateral rotatory olisthesis with neural canal dimensions. Spine (Phila Pa 1976) 2006;31:2353-8.

[26] Ploumis A, Liu H, Mehbod AA, et al. A correlation of radiographic and functional measurements in adult degenerative scoliosis. Spine (Phila Pa 1976) 2009;34:1581-4.

[27] Humbert L, De Guise JA, Aubert B, et al. 3D reconstruction of the spine from biplanar X-rays using parametric models based on transversal and longitudinal inferences. Med Eng Phys 2009;31:681-7.

[28] Ferrero E, Lafage R, Vira S, et al. Three-dimensional reconstruction using stereoradiography for evaluating adult spinal deformity: a reproducibility study. Eur Spine J 2016. doi: 10.1007/s00586-0164833-5 [Epub ahead of print].

[29] Sangole A, Aubin C-E, Labelle H, et al. The central hip vertical axis: a reference axis for the Scoliosis Research Society three-dimensional classification of idiopathic scoliosis. Spine (Phila Pa 1976) 2010;35: E530-4.

[30] Protopsaltis T, Schwab F, Smith JS, et al. International Spine Study Group. The T1 pelvic angle (TPA), a novel radiographic measure of sagittal deformity, accounts for both pelvic retroversion and truncal inclination and correlates strongly with HRQOL. J Bone Joint Surg Am 2014;96:1631-40.

[31] Terran J, Schwab F, Shaffrey CI, et al. The SRS-Schwab adult spinal deformity classification: assessment and clinical correlations based on a prospective operative and nonoperative cohort. Neurosurgery 2013;73:559-68. 
[32] Schwab F, Ungar B, Blondel B, et al. Scoliosis Research SocietySchwab adult spinal deformity classification: a validation study. Spine (Phila Pa 1976) 2012;37:1077-82.

[33] Cobb JR. Progress in orthopedic surgery for 1945; conditions involving the spine and thorax, exclusive of those in the lower part of the back. Arch Surg 1949;55:76-87.

[34] Nash CLJ, Moe JH. A study of vertebral rotation. J Bone Joint Surg Am 1969;51:223-9.

[35] Lam GC, Hill DL, Le LH, et al. Vertebral rotation measurement: a summary and comparison of common radiographic and CT methods. Scoliosis 2008;3:16.

[36] Weiss HR. Measurement of vertebral rotation: Perdriolle versus Raimondi. Eur Spine J 1995;4:34-8.
[37] Nault M-L, Mac-Thiong J-M, Roy-Beaudry M, et al. Three-dimensional spinal morphology can differentiate between progressive and non-progressive patients with adolescent idiopathic scoliosis at the initial presentation. Spine (Phila Pa 1976) 2014;39:E601-6.

[38] Villemure I, Aubin CE, Grimard G, et al. Progression of vertebral and spinal three-dimensional deformities in adolescent idiopathic scoliosis: a longitudinal study. Spine (Phila Pa 1976) 2001;26: 2244-50

[39] Trammell TR, Schroeder RD, Reed DB. Rotatory olisthesis in idiopathic scoliosis. Spine (Phila Pa 1976) 1988;13:1378-82.

[40] Marty-Poumarat C, Scattin L, Marpeau M, et al. Natural history of progressive adult scoliosis. Spine (Phila Pa 1976) 2007;32: 1227-34. 